Original Research Paper

\title{
A New Extension of the Lomax Distribution with Statistical Properties and Applications to Failure and Service Times Data Sets
}

\author{
Mohamed K.A. Refaie \\ Agami High Institute of Administrative Sciences, Alexandria, Egypt
}

Article history

Received: 16-11-2018

Revised: $28-12-2018$

Accepted: 01-02-2019

Email: refaie_top@yahoo.com

\begin{abstract}
In this work, we introduce and study a new alternative Lomax model. The maximum likelihood method is used to estimate the unknown model parameters. We show empirically the importance and wide flexibility of the new model in modeling two types of failure times data sets. The new model is much better than the gamma Lomax, exponentiated Lomax, beta Lomax and Lomax models so the new model is a good alternative to these models.
\end{abstract}

Keywords: Lomax Model, Odd Lindley-G Family, Estimation

\section{Introduction and Justification}

A random variable (r.v.) $Z$ has the exponentiated Lomax $\left(\mathrm{EL}_{x}\right)$ distribution with three parameters $\alpha$ (power parameter), $\lambda$ and $\beta$, if its Cumulative Distribution Function (CDF) is given by:

$$
\left.\Pi_{\alpha, \lambda, \beta}(z)\right|_{(z>0)}=\left[1-\left(1+z \beta^{-1}\right)^{-\lambda}\right]^{\alpha}
$$

where, $\alpha>0, \lambda>0$ and $\beta>0$ are the shape parameters. Then the corresponding Probability Density Function (PDF) of (1) is:

$$
\left.\pi_{\alpha, \lambda, \beta}(z)\right|_{(z>0)}=\alpha \lambda \beta^{-1}\left(1+z \beta^{-1}\right)^{-(1+\lambda)}\left[1-\left(1+z \beta^{-1}\right)^{-\lambda}\right]^{\alpha-1},
$$

when $\alpha=1$ we get the Lomax $\left(\mathrm{L}_{x}\right)$ or the Pareto type II (PaII) model with:

$$
G_{\lambda, \beta}(z)=1-\left(1+z \beta^{-1}\right)^{-\lambda} \text { and } g_{\lambda, \beta}(z)=\lambda \beta^{-1}\left(1+z \beta^{-1}\right)^{-(1+\lambda)}
$$

The $\mathrm{L}_{x}$ model (Lomax, 1954) was originally pioneered for modeling business failure data. The $\mathrm{L}_{x}$ distribution has been found a wide application in many fields such as engineering, biological sciences, actuarial science, size of cities, income studies, wealth inequality, medical and reliability modeling. It has been applied for modeling data obtained from income and wealth (Harris, 1968; Atkinson and Harrison, 1978), reliability and life testing (Hassan and Al-Ghamdi, 2009), Hirsch related statistics (Glanzel, 2008), for modeling gauge lengths data
(Afify et al., 2015), for modeling bladder cancer patient's data and remission times data (Yousof et al., 2017).

The main goal of this article is to introduce a new $L_{x}$ model using the Odd Lindley-G (OLi-G) family of distributions (Silva et al., 2017) with scale parameter $a=$ 1, the PDF and CDF of the OLi-G family of distribution are respectively given by:

$$
f_{\psi}\left(x ;\left.a\right|_{(a=1)}\right)=\frac{1}{2} \pi_{\psi}(x) \bar{\Pi}_{\psi}(x)^{-3} \exp \left[-\frac{\Pi_{\psi}(x)}{\bar{\Pi}_{\psi}(x)}\right],
$$

and:

$$
\begin{aligned}
& F_{\psi}(x ; a \mid a=1) \\
& =1-\left[2+\bar{\Pi}_{\psi}(x)\right]\left[2 \bar{\Pi}_{\psi}(x)\right]^{-1} \exp \left[-\frac{\Pi_{\psi}(x)}{\bar{\Pi}_{\psi}(x)}\right],
\end{aligned}
$$

where, $\Pi_{\psi}(x)$ is the baseline $\mathrm{CDF}, \psi=(\alpha, \lambda, \beta)$ is the parameter vector of the baseline distribution and $\bar{\Pi}(x, \psi)=$ $1-\Pi(x, \psi)$ is the Survival Function (SF) of the baseline distribution. To this end, we use Equations (1), (2) and (3) to obtain the new three-parameter $\mathrm{OLiEL}_{x} \operatorname{PDF}($ for $x>0$ ):

$$
\begin{aligned}
& f_{\alpha, \lambda, \beta}(x)=\frac{1}{2} \alpha \lambda \beta^{-1}\left(1+x \beta^{-1}\right)^{-(1+\lambda)}\left[1-\left(1+x \beta^{-1}\right)^{-\lambda}\right]^{\alpha-1} \\
& \times\left\{1-\left[1-\left(1+x \beta^{-1}\right)^{-\lambda}\right]^{\alpha}\right\}^{-3} \exp \left\{-\frac{\left[1-\left(1+x \beta^{-1}\right)^{-\lambda}\right]^{\alpha}}{1-\left[1-\left(1+x \beta^{-1}\right)^{-\lambda}\right]^{\alpha}}\right\},
\end{aligned}
$$

the corresponding CDF to (5) is given by: 


$$
F_{\alpha, \lambda, \beta}(x)=1-\left[\begin{array}{c}
\left(2+\left\{1-\left[1-\left(1+x \beta^{-1}\right)^{-\lambda}\right]^{\alpha}\right\}\right) \\
\times\left(2\left\{1-\left[1-\left(1+x \beta^{-1}\right)^{-\lambda}\right]^{\alpha}\right\}\right)^{-1} \\
\times \exp \left\{-\frac{\left[1-\left(1+x \beta^{-1}\right)^{-\lambda}\right]^{\alpha}}{1-\left[1-\left(1+x \beta^{-1}\right)^{-\lambda}\right]^{\alpha}}\right\},
\end{array}\right] .
$$

when $\alpha=1$ we get the two-parameter $\mathrm{OLiL}_{x}$ model (Silva et al., 2017). The Hazard Rate Function (HRF) of the $\mathrm{OLiEL}_{x}$ distribution can be obtained by as:
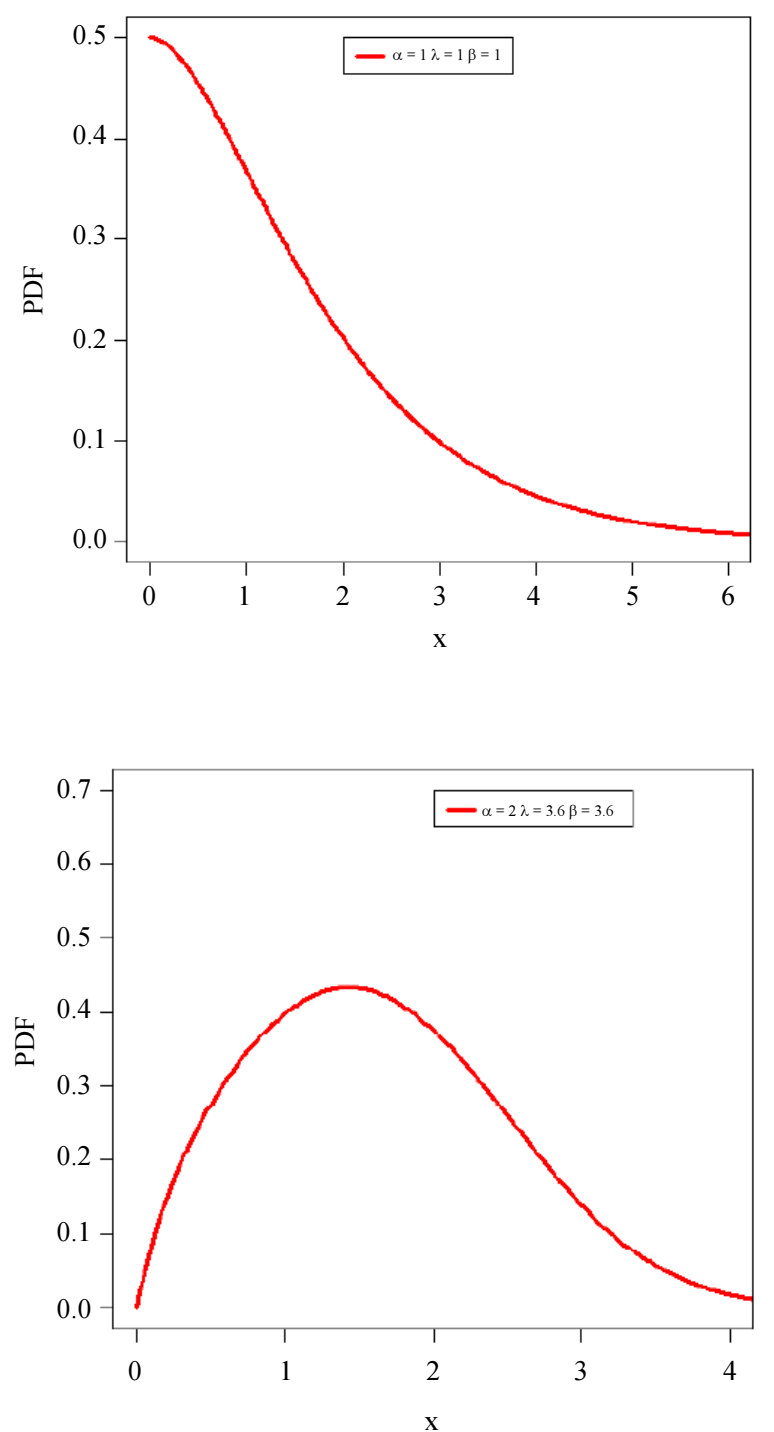

$$
\begin{aligned}
& h_{\alpha, \lambda, \beta}(x)=\frac{1}{2} \alpha \lambda \beta^{-1}\left(1+x \beta^{-1}\right)^{-(1+\lambda)} \\
& \times\left(\frac{2+\left\{1-\left[1-\left(1+x \beta^{-1}\right)^{-\lambda}\right]^{\alpha}\right\}}{2\left\{1-\left[1-\left(1+x \beta^{-1}\right)^{-\lambda}\right]^{\alpha}\right\}}\right)^{-1} \\
& \times\left[1-\left(1+x \beta^{-1}\right)^{-\lambda}\right]^{\alpha-1}\left\{1-\left[1-\left(1+x \beta^{-1}\right)^{-\lambda}\right]^{\alpha}\right\}^{-3} .
\end{aligned}
$$

We draw the PDF and HRF plots of the OLiEL $x$ distribution in Fig. 1 and 2 for selected parameters values. We see that its PDF can be unimodal and decreasing. Also, its HRF can be only increasing.
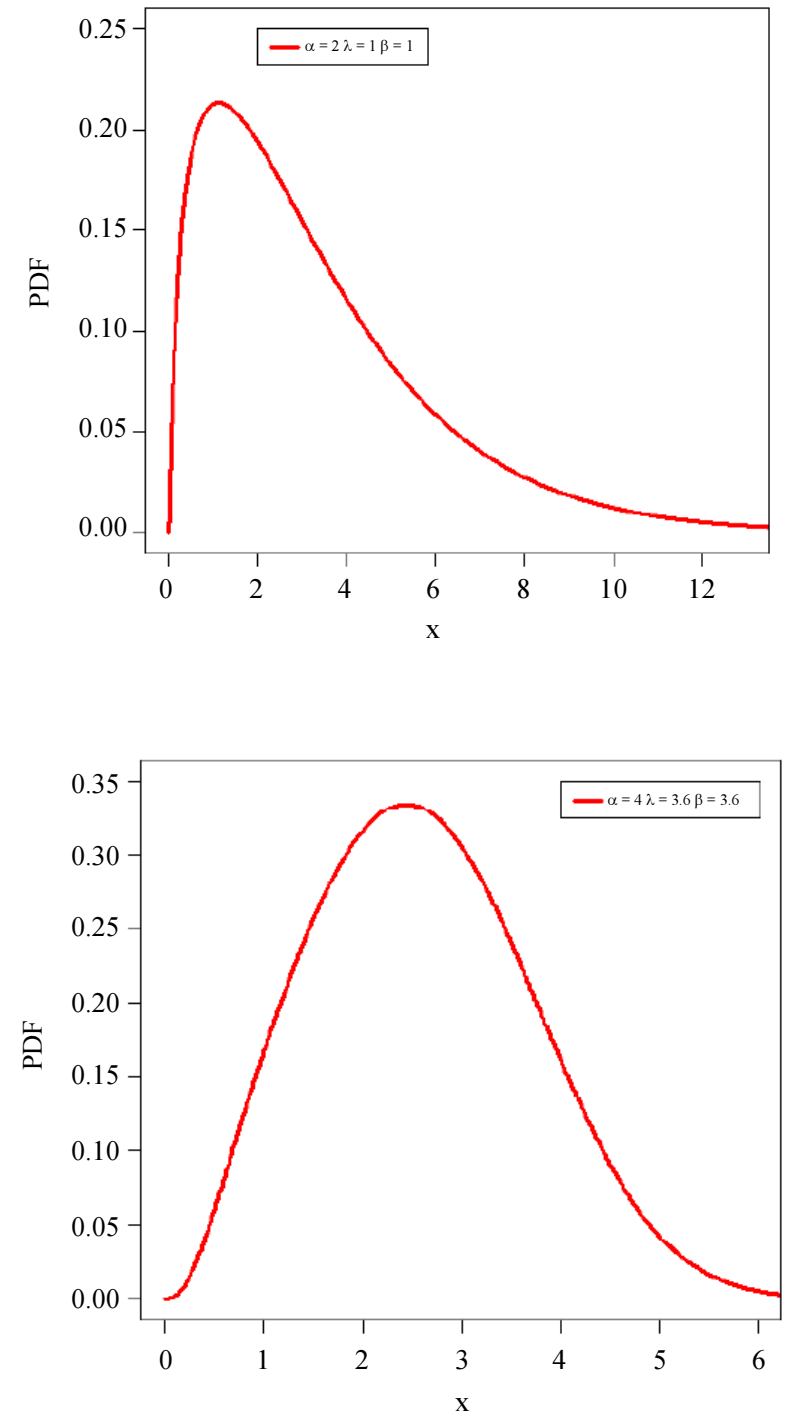

Fig. 1: Plots of the OLiELx PDF 

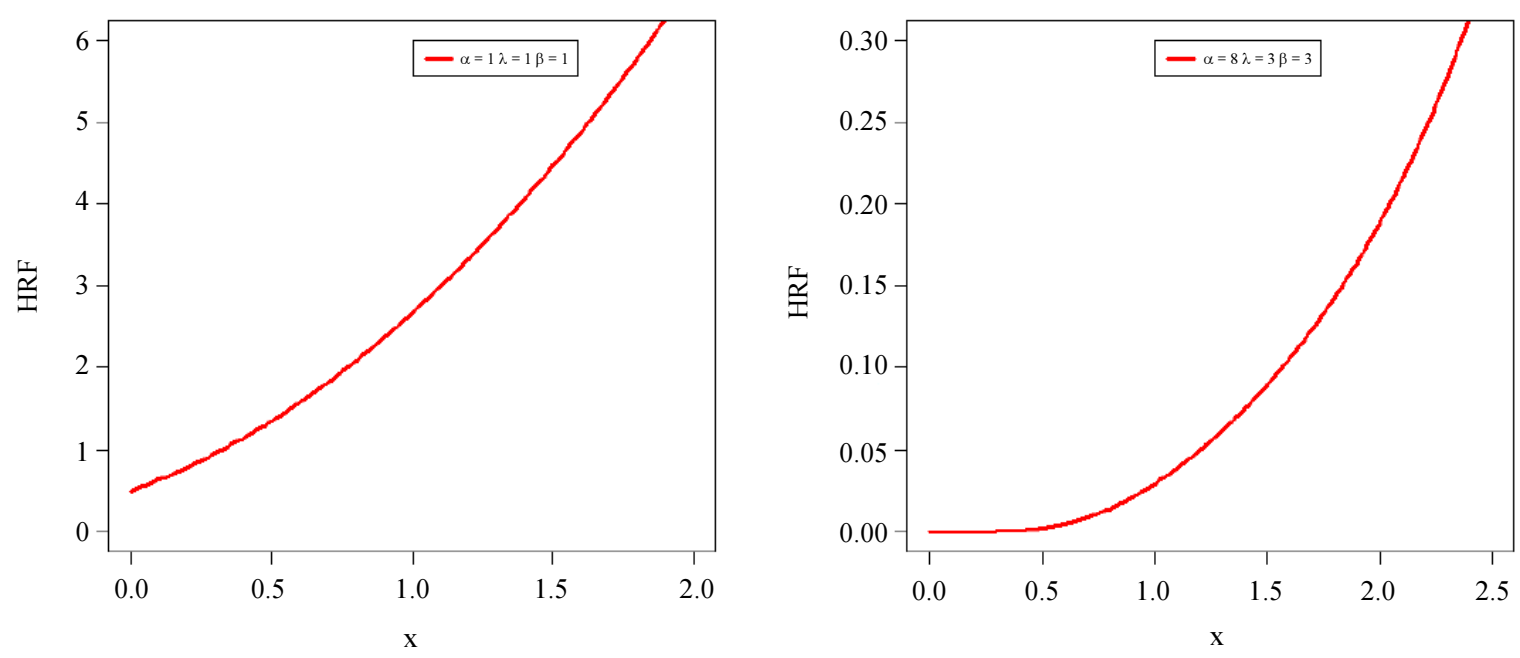

Fig. 2: Plots of the $\mathrm{OLiEL}_{x} \mathrm{HRF}$

The major justification for the practicality of the new model is based on the enormous use of the $\mathrm{EL}_{x}$ and $\mathrm{L}_{x}$ models in modeling failure times data sets. The OLiEL $L_{x}$ is a good alternative to the $\mathrm{EL}_{x}$, the gamma Lomax, the beta Lomax, $\mathrm{L}_{x}$ models as illustrated using two real data sets. The $\mathrm{OLiEL}_{x}$ can also be considered as an appropriate model for fitting the right skewed and the unimodal data sets (see applications 1 and 2).

The rest of the paper is outlined as follows. In section 2, we derive some statistical properties for the new model. Maximum likelihood estimation of the model parameters is addressed in section 3. Section 4 gives the simulation studies. We provide two applications to real data sets to illustrate the importance of the new model in Section 4. Finally, we offer some concluding remarks in Section 5.

\section{Statistical Properties}

\section{Shapes}

The critical points of the $\mathrm{OLiEL}_{x}$ density function are the roots of the equation:

$$
\begin{aligned}
& 0=3 \alpha \lambda \beta^{-1}\left(1+x \beta^{-1}\right)^{-(1+\lambda)}\left[1-\left(1+x \beta^{-1}\right)^{-\lambda}\right]^{\alpha-1}\left\{1-\left[1-\left(1+x \beta^{-1}\right)^{-\lambda}\right]^{\alpha}\right\}^{-1} \\
& +\alpha \lambda \beta^{-1}\left(1+x \beta^{-1}\right)^{-(1+\lambda)}\left[1-\left(1+x \beta^{-1}\right)^{-\lambda}\right]^{\alpha-1}\left\{1-\left[1-\left(1+x \beta^{-1}\right)^{-\lambda}\right]^{\alpha}\right\}^{-2} \\
& +\left(\begin{array}{c}
\beta(\alpha \lambda)^{-1}\left(1+x \beta^{-1}\right)^{1+\lambda}\left[1-\left(1+x \beta^{-1}\right)^{-\lambda}\right]^{1-\alpha} \\
\times \frac{d}{d x}\left\{\alpha \lambda \beta^{-1}\left(1+x \beta^{-1}\right)^{-(1+\lambda)}\left[1-\left(1+x \beta^{-1}\right)^{-\lambda}\right]^{\alpha-1}\right\}
\end{array}\right) .
\end{aligned}
$$

The critical points of the of the HRF of the OLiEL $x$ are obtained from the following equation:

$$
\begin{aligned}
& 0=\alpha \lambda \beta^{-1}\left(1+x \beta^{-1}\right)^{-(1+\lambda)}\left[1-\left(1+x \beta^{-1}\right)^{-\lambda}\right]^{\alpha-1}\left\{2-\left[1-\left(1+x \beta^{-1}\right)^{-\lambda}\right]^{\alpha}\right\} \\
& +2 \alpha \lambda \beta^{-1}\left(1+x \beta^{-1}\right)^{-(1+\lambda)}\left[1-\left(1+x \beta^{-1}\right)^{-\lambda}\right]^{\alpha-1}\left\{1-\left[1-\left(1+x \beta^{-1}\right)^{-\lambda}\right]^{\alpha}\right\}^{-2} \\
& +\left(\begin{array}{c}
(\alpha \lambda)^{-1} \beta\left(1+x \beta^{-1}\right)^{1+\lambda}\left[1-\left(1+x \beta^{-1}\right)^{-\lambda}\right]^{1-\alpha} \\
\frac{d}{d x}\left\{\alpha \lambda \beta^{-1}\left(1+x \beta^{-1}\right)^{-(1+\lambda)}\left[1-\left(1+x \beta^{-1}\right)^{-\lambda}\right]^{\alpha-1}\right\}
\end{array}\right.
\end{aligned}
$$

We can examine the last two Equations to determine the local maximums and minimums and inflexion points via most computer algebra systems.

\section{Quantile Function}

Let $X$ be a r.v. with $\operatorname{CDF} F_{\lambda, \beta}(x)$. For any $u \in(0.1)$, the $u^{\text {th }}$ Quantile Function (QF) $Q(u)$ of the r.v. $X$ is the solution of $u=F(Q(u))$ for all $Q(u)>0$, from Equation (6), we get:

$$
(u-1) 2 \exp (2)=-\frac{2-G(Q(u))}{1-G(Q(u))} \exp \left\{-\frac{2-G(Q(u))}{1-G(Q(u))}\right\},
$$

where:

$$
-\frac{2-G(Q(u))}{1-G(Q(u))}
$$

is the Lambert $W(\cdot)$ function of the real argument $2(u-1)$ exp (2): From Silva et al. (2017), we can write the following equation for $\mathrm{QF}$ of the new model as:

$$
Q(u)=\beta\left[\left(1-\left\{1-a[1+W(2(u-1) \exp (2))]^{-1}\right\}^{\frac{1}{\alpha}}\right)^{-\frac{1}{\lambda}}-1\right],
$$


where, $W(\cdot)$ is Lambert function.

\section{Useful Expansions}

Starting with the PDF in (5) and using the power series for the exponential function:

$$
\exp \left\{-\frac{\left[1-\left(1+x \beta^{-1}\right)^{-\lambda}\right]^{\alpha}}{1-\left[1-\left(1+x \beta^{-1}\right)^{-\lambda}\right]^{\alpha}}\right\}
$$

we have:

$$
\begin{aligned}
& f_{\alpha, \lambda, \beta}(x)=\frac{1}{\frac{1}{2} \alpha, \lambda, \beta^{-1}\left(1+x \beta^{-1}\right)^{-(1+\lambda)}\left[1-\left(1+x \beta^{-1}\right)^{-\lambda}\right]^{\alpha-1}} \\
& \times \sum_{k=0}^{\infty} \frac{(-1)^{k}}{k !} \overbrace{\left[\left[1-\left(1+x \beta^{-1}\right)^{-\lambda}\right]^{\alpha}\right\}^{k}}^{\bar{\pi}_{\alpha, \lambda, \beta}(x)} \\
& \times \underbrace{\left.\left\{1-\left[1-\left(1+x \beta^{-1}\right)^{-\lambda}\right]^{-(3+k)}\right\}^{\alpha}\right\}^{-(3+k)}}_{\left[1-\Pi_{\alpha, \lambda, \beta}(x)\right]^{k}},
\end{aligned}
$$

Using the generalized binomial expansion, we can write:

$$
\begin{aligned}
& \left\{1-\left[1-\left(1+x \beta^{-1}\right)^{-\lambda}\right]^{\alpha}\right\}^{-(k+3)} \\
& =\sum_{m=0}^{\infty} \frac{\Gamma(3+m+k)}{m ! \Gamma(3+k)}\left\{\left[1-\left(1+x \beta^{-1}\right)^{-\lambda}\right]^{\alpha}\right\}^{m},
\end{aligned}
$$

then the $\mathrm{OLiEL}_{x}$ density function can be expressed as an infinite mixture of exponentiated- $\mathrm{L}_{x}\left(\mathrm{EL}_{x}\right)$ density functions:

$$
f_{\alpha, \lambda, \beta}(x)=\sum_{m, k=0}^{\infty} v_{m, k} \pi_{[(1+m+k) \alpha+1], \lambda, \beta}(x)
$$

where:

$$
v_{m, k}=\frac{(-1)^{k} \Gamma(3+m+k)}{m ! k !(1+\lambda)[(1+m+k) \alpha+1] \Gamma(3+k)}
$$

and:

$$
\begin{aligned}
& \pi_{[(1+m+k) \alpha+1], \lambda, \beta}(x)=[(1+m+k) \alpha+1] \\
& \times \underbrace{\times\left[1-\left(1+x \beta^{-1}\right)^{-\lambda}\right]^{-(1+m+k) \alpha}}_{\Pi_{(1+m+k) \alpha, \lambda, \beta}(x)} \\
& \times \underbrace{\times \alpha \lambda \beta^{-1}\left(1+x \beta^{-1}\right)^{-(1+\lambda)}\left[1-\left(1+x \beta^{-1}\right)^{-\lambda}\right]^{\alpha-1}}_{\pi_{\alpha, \lambda, \beta}(x)}
\end{aligned}
$$

is the $\mathrm{EL}_{x}$ density with power parameter $[(1+m+k) \alpha$ +1 ]. Similarly:

$$
F_{\alpha, \lambda, \beta}(x)=\sum_{m, k=0}^{\infty} v_{m, k} \Pi_{[(1+m+k) \alpha+1], \lambda, \beta}(x),
$$

\section{Moments}

The $r^{(t h)}$ ordinary moment of $X$ is given by:

$$
\mu_{r}^{\prime}=E\left(X^{r}\right)=\sum_{m, k=0}^{\infty} v_{m, k} \int_{0}^{\infty} x^{r} \pi_{[(1+m+k) \alpha+1], \lambda, \beta}(x) d x,
$$

then we obtain:

$$
\mu_{r}^{\prime}=\left.\sum_{m, k=0}^{\infty} \sum_{w=0}^{r} v_{m, k, w}^{(r,[(1+m+k) \alpha+1])} B\left(\begin{array}{l}
{[(1+m+k) \alpha+1]} \\
1+(w-r) / \lambda
\end{array}\right)\right|_{(r \leq \lambda)}
$$

where:

$$
B(c ; d)=\int_{0}^{1} u^{c-1}(1-u)^{d-1} d u=\Gamma(c) \Gamma(d) /\left.\Gamma(c+d)\right|_{(c, d \notin(0,-1,-2, \ldots))}
$$

is the complete beta function and:

$$
v_{m, k, w}^{(r,[(1+m+k) \alpha+1])}=v_{m, k}([(1+m+k) \alpha+1]) \beta^{r}(-1)^{w}\left(\begin{array}{l}
r \\
w
\end{array}\right) .
$$

Setting $r=1,2,3$ and 4 in (9) we get:

$$
E(X)=\mu_{1}^{\prime}=\sum_{m, k=0}^{\infty} \sum_{w=0}^{r} v_{m, k, w}^{(1,[(1+m+k) \alpha+1])} B([(1+m+k) \alpha+1]), 1+(w-1) /\left.\lambda\right|_{(1 \leq \lambda)},
$$

which is the mean of $X$ :

$$
\begin{aligned}
& E\left(X^{2}\right)=\mu_{2}^{\prime}=\sum_{m, k=0}^{\infty} \sum_{w=0}^{r} v_{m, k, w}^{(2,[(1+m+k) \alpha+1])} B([(1+m+k) \alpha+1]), 1+(w-2) /\left.\lambda\right|_{(2 \leq \lambda)}, \\
& E\left(X^{3}\right)=\mu_{3}^{\prime}=\sum_{m, k=0}^{\infty} \sum_{w=0}^{r} v_{m, k, w}^{(3,[(1+m+k) \alpha+1])} B([(1+m+k) \alpha+1]), 1+(w-3) /\left.\lambda\right|_{(3 \leq \lambda)}, \\
& E\left(X^{4}\right)=\mu_{4}^{\prime}=\sum_{m, k=0}^{\infty} \sum_{w=0}^{r} v_{m, k, w}^{(4,[(1+m+k) \alpha+1])} B([(1+m+k) \alpha+1]), 1+(w-4) /\left.\lambda\right|_{(4 \leq \lambda)},
\end{aligned}
$$


The last four equations can be used for obtaining the first four moments about the mean. In general the $r^{\text {(th) }}$ central moment of $X$, say $M_{r}$, is:

$$
\mu_{r}=E(X-\mu)^{r}=\sum_{h=0}^{r}(-1)^{h}\left(\begin{array}{l}
r \\
h
\end{array}\right)\left(\mu_{1}^{\prime}\right)^{r} \mu_{r-h}^{\prime},
$$

where, $\mu=E(X)$. The skewness $\left(\sqrt{\beta_{1}}\right)$ and kurtosis $\left(\beta_{2}\right)$ measures also can be calculated from the ordinary moments using well-known relationships. For the skewness and kurtosis coefficients, we have:

$$
\sqrt{\beta_{1}}=\sqrt{\frac{\mu_{3}^{3}}{\mu_{2}^{3}}} \text { and } \beta_{2}=\frac{\mu_{4}}{\mu_{2}^{2}},
$$

respectively.

We prove numerically that the new distribution provides better fits to two real data sets than other four extended $L_{x}$ models with two, three and four parameters (see Section 4). These two examples show that the new $\mathrm{OLiEL}_{x}$ distribution is a good alternative for modeling failure times data. Further, the $\mathrm{OLiEL}_{x}$ density can be right-skewed or symmetric (Fig. 1). Whereas the $\mathrm{OLiEL}_{x}$ HRF can be monotonically increasing (Fig. 2). The $\sqrt{\beta_{1}}$ of the $\mathrm{OLiEL}_{x}$ distribution can range in the interval ($2.106,4.424)$, whereas the $\beta_{2}$ of the $\mathrm{OLiEL}_{x}$ distribution varies only in the interval $(-14,38)$ (Table 1$)$.

\section{Generating Function}

The moment generating function (mgf) $M_{X}(t)=$ $E\left(e^{t X}\right)$ can be derived from Equation (7) as follows:

$$
\begin{aligned}
& M_{X}(t)=\sum_{m, k, r=0}^{\infty} \sum_{w=0}^{r} \frac{t^{r}}{r !} v_{m, k, w}^{(r,[(1+m+k) \alpha+1])} B([(1+m+k) \alpha+1]), \\
& 1+(w-r) /\left.\lambda\right|_{(r \leq \lambda)},
\end{aligned}
$$

\section{Incomplete Moments and Mean Deviations}

The $s^{(t h)}$ incomplete moment, say $I_{s}(t)$, of $X$ can be expressed from (7) as:

$$
I_{s}(t)=\left.\sum_{m, k=0}^{\infty} \sum_{w=0}^{r} v_{m, k, w}^{(s,[(1+m+k) \alpha+1])} B_{t}(m+k+1,1+(w-s) / \lambda)\right|_{(s \leq \lambda)},
$$

where:

$$
B_{t}(p ; q)=\int_{0}^{t} u^{p-1}(1-u)^{q-1} d u=\sum_{j=0}^{\infty} \frac{(1-q)_{j}}{j !(p+j)} t^{p+j}
$$

is the incomplete beta function and:

$$
(n)_{k}=n(n+1) \ldots(n+k-1)
$$

is known as Pochhammer's symbol after the German mathematician Pochhammer [1841-1920].

The mean deviations about the mean:

$$
\begin{aligned}
M D_{(E(X))} & =E(|X-E(X)|) \\
& =-2 I_{1} E(X)+2 E(X) F(E(X))
\end{aligned}
$$

and about the median:

$$
\begin{aligned}
M D_{(\operatorname{Median}(X))} & =E(|X-\operatorname{Median}(X)|) \\
& =-2 I_{1}(\operatorname{Median}(X))+E(X)
\end{aligned}
$$

of $X, F(E(X))$ is easily calculated from (5) and $I_{1}(t)$ is the first incomplete moment given by the last Equation with $s=$ 1. A general equation for $I_{1}(t)$ can be derived from $I_{s}(t)$ as:

$$
\begin{aligned}
& I_{1}(t) \\
& =\sum_{m, k=0}^{\infty} \sum_{w=0}^{r} v_{m, k, w}^{(1,[(1+m+k) \alpha+1])} B_{t}([(1+m+k) \alpha+1], 1+(w-1) / \lambda) .
\end{aligned}
$$

\section{Moment of Residual and Reversed Residual Life}

The $n^{(t h)}$ moment of the residual life, say:

$$
m_{n}(t)=E\left[\left.(X-t)^{n}\right|_{(X>t, n=1,2, \ldots)}\right],
$$

uniquely determines $F(x)$. The $n^{(t h)}$ moment of the residual life of $X$ is given by:

$$
m_{n}(t)=\left[1-F_{\alpha, \lambda, \beta}(t)\right]^{-1} \int_{t}^{\infty}(x-t)^{n} d F_{\alpha, \lambda, \beta}(x)
$$

therefore:

$$
\begin{aligned}
& m_{n}(t) \\
& =\left.\sum_{m, k=0}^{\infty} \sum_{w=0}^{s} \frac{a_{m, k, w}^{(n,[(1+m+k) \alpha+1])}}{1-F_{\lambda, \beta}(t)} B_{t}([(1+m+k) \alpha+1], 1+(w-n) / \lambda)\right|_{(n \leq \lambda)},
\end{aligned}
$$

where:

$$
a_{m, k, w}^{(n,[(1+m+k) \alpha+1])}=v_{m, k, w}^{(n,[(1+m+k) \alpha+1])} \sum_{d=0}^{n}(1-t)^{n} .
$$

The $n^{(t h)}$ moment of the reversed residual life, say:

$$
M_{n}(t)=E\left[\left.(t-X)^{n}\right|_{(X \leq t, t>0, n=1,2, \ldots)}\right],
$$

uniquely determines $F(x)$. We obtain:

$$
M_{n}(t)=F_{\alpha, \lambda, \beta}(t)^{-1} \int_{0}^{t}(t-x)^{n} d F_{\alpha, \lambda, \beta}(x) .
$$


Table 1: Mean, variance, skewness and kurtosis of the $\mathrm{OLiEL}_{x}$ distribution with $\beta=1.5$ and different values of $\alpha$ and $\lambda$

\begin{tabular}{llllll}
\hline$\lambda$ & $\alpha$ & Mean & Variance & $\sqrt{\beta_{1}}$ & $\beta_{2}$ \\
\hline 0.5 & 0.5 & 2.872888 & 17.1728 & 3.784633 & 29.29589 \\
& 1 & 10.5 & 222.7499 & 4.02216 & 33.08947 \\
& 2 & 38.44636 & 3137.958 & 4.223212 & 36.22753 \\
& 5 & 222.8358 & 112857.4 & 4.370628 & 38.57605 \\
& 10 & 864.9294 & 1755134 & 4.423885 & -14.07279 \\
& 20 & 3404.382 & 27681971 & -2.105726 & 2.763589 \\
2.5 & 0.5 & 0.2794296 & 0.0629023 & 1.179014 & 4.319735 \\
& 1 & 0.6025587 & 0.1720848 & 0.7994452 & 3.377934 \\
& 2 & 1.104976 & 0.3774538 & 0.597283 & 3.064221 \\
& 5 & 2.109887 & 0.9002299 & 0.4757101 & 2.940227 \\
& 10 & 3.198071 & 1.638229 & 0.4364157 & 2.910089 \\
5 & 4.656197 & 2.915106 & 0.4172134 & 2.896965 \\
& 0.5 & 0.1299453 & 0.01242288 & 1.007976 & 3.682173 \\
& 1.5 & 0.3752224 & 0.04227459 & 0.4161971 & 2.681674 \\
& 2.5 & 0.5382854 & 0.06152505 & 0.2757932 & 2.607453 \\
& 5 & 0.8070189 & 0.09249494 & 0.1645138 & 2.596024 \\
& 10 & 1.129978 & 0.1303217 & 0.1072898 & 2.606683 \\
& 20 & 1.509419 & 0.177695 & 0.07841155 & 2.616292 \\
\hline
\end{tabular}

Then, the $n^{(t h)}$ moment of the reversed residual life of $X$ becomes:

$$
M_{n}(t)=\left.\sum_{m, k=0}^{\infty} \sum_{w=0}^{s} \frac{c_{m, k, w}^{(n,[(1+m+k) \alpha+1])}}{F_{\alpha, \lambda, \beta}(t)} B_{t}\left(\begin{array}{l}
{[(1+m+k) \alpha+1]} \\
1+(w-n) / \lambda
\end{array}\right)\right|_{(n \leq \lambda)},
$$

where:

$$
c_{m, k, w}^{(n,[(1+m+k) \alpha+1])}=v_{m, k, w}^{(n,[(1+m+k) \alpha+1])} \sum_{d=0}^{n}(-1)^{d}\left(\begin{array}{l}
n \\
d
\end{array}\right) t^{n-d} .
$$

\section{Order Statistics}

Let $X_{1}, \ldots, X_{n}$ be a random sample from the $\mathrm{OLiEL}_{x}$ model of distributions and let $X_{1: n}, \ldots, X_{n: n}$ be the corresponding order statistics. The PDF of the $i^{(t h)}$ order statistic, say $X_{i: n}$, can be expressed as:

$$
\begin{aligned}
& f_{\alpha, \lambda, \beta}^{(i: n)}(x)=[B(i, n-i+1)]^{-1} \\
& f_{\alpha, \lambda, \beta}(x) F_{\alpha, \lambda, \beta}(x)^{i-1}\left[1-F_{\alpha, \lambda, \beta}(x)\right]^{n-i},
\end{aligned}
$$

where, $B(\cdot \cdot)$ is the beta function. Substituting (5) and (6) in Equation (10), we obtain:

$$
f_{\alpha, \lambda, \beta}^{(i, n)}(x)=\sum_{m, p=0}^{\infty} \sum_{j=0}^{k+n-i} \Upsilon_{m, p, j} \pi_{[(j+m+p) \alpha+1], \lambda, \beta}(x)
$$

where:

$$
\begin{aligned}
& \Upsilon_{m, p, j}=\sum_{k=0}^{i=1} \frac{(-1)^{k+m} \lambda^{j+m+2}(1+\lambda)^{-(j+1)}}{m ! B(i, n-i+1)[(j+m+p) \alpha+1]} \\
& \left(\begin{array}{c}
(j+m+p) \alpha \\
j+m
\end{array}\right)\left(\begin{array}{c}
k+n-1 \\
j
\end{array}\right)\left(\begin{array}{c}
i-1 \\
k
\end{array}\right) .
\end{aligned}
$$

Then, the $q^{\text {th }}$ moment of $X_{i: n}$ is given by $(\forall \lambda>q)$ :

$$
\begin{aligned}
& E\left(X_{i: n}^{q}\right)=\sum_{m, p=0}^{\infty} \sum_{j=0}^{k+n-i} \sum_{w=0}^{r} \Upsilon_{m, k, j, w}^{(q[(j+m+p-1) \alpha+1])} \\
& B([(j+m+p-1) \alpha+1], 1+(w-q) / \lambda),
\end{aligned}
$$

where:

$$
\Upsilon_{m, k, j, w}^{(q,[(j+m+p-1) \alpha+1])} \Upsilon_{m, p, j}[(j+m+p) \alpha+1] \beta^{q}(-1)^{w}\left(\begin{array}{l}
q \\
w
\end{array}\right)
$$

\section{Estimation}

The Maximum Likelihood Estimators (MLEs) enjoy desirable properties and can be used for constructing confidence intervals and regions and also in test statistics. Let $x_{1}, \ldots, x_{n}$ be a random sample from $\mathrm{OLiEL}_{x}$ distribution with parameters $\alpha, \lambda$ and $\beta$. Let $\Psi=(\alpha, \lambda, \beta)^{\mathrm{T}}$ be the $3 \times 1$ parameter vector. For determining the MLE of $\Psi$, we have the log-likelihood function:

$$
\begin{aligned}
& \ell=\ell(\Psi)=-n \log 2+n \log \alpha+n \log \lambda \\
& -n \log \beta-(1+\lambda) \sum_{i=1}^{n} \log \left(1+x_{i} \beta^{-1}\right) \\
& +(\alpha-1) \sum_{i=1}^{n} \log \left[1-\left(1+x_{i} \beta^{-1}\right)^{-\lambda}\right] \\
& -3 \sum_{i=1}^{n} \log \left\{1-\left[1-\left(1+x_{i} \beta^{-1}\right)^{-\lambda}\right]^{\alpha}\right\} \\
& -\sum_{i=1}^{n}\left(\left[1-\left(1+x_{i} \beta^{-1}\right)^{-\lambda}\right]^{\alpha} /\left\{1-\left[1-\left(1+x_{i} \beta^{-1}\right)^{-\lambda}\right]^{\alpha}\right\}\right) .
\end{aligned}
$$

The components of the score vector: 


$$
U(\Psi)=\frac{\partial \ell(\Psi)}{\partial \Psi}=\left(\frac{\partial \ell(\Psi)}{\partial \alpha}, \frac{\partial \ell(\Psi)}{\partial \lambda}, \frac{\partial \ell(\Psi)}{\partial \beta}\right)^{\mathrm{T}},
$$

can be easily derived.

\section{Simulation Studies}

We consider a random sample of size $\mathrm{n}=20,50,100$, 150, 500 and 1000 from the $\mathrm{OliEL}_{x}$ density corresponding to particular choices of the parameters:

- $\quad$ I: $\alpha=0.5, \lambda=1.5, \beta=0.5$

- $\mathrm{I}: \alpha=0.5, \lambda=3, \beta=1.5$

The results are presented in Table 2. We provide the MLEs, biases (Bias) and Mean Square of Errors (MSEs) for the estimates of all the parameters under both the methods of estimation. The results show that the maximum likelihood estimation performs well. In general, the biases and MSEs of the parameters are reasonably small. The biases and MSEs decrease as the sample size increases. The results suggest that the ML method can be used to estimate the parameters of the $\mathrm{OLiEL}_{x}$ model.

\section{Real Data Modeling}

In this section, we provide two applications to two real data sets to prove the importance and flexibility of the $\mathrm{OLiEL}_{x}$ distribution. We compare the fit of the $\mathrm{OLiEL}_{x}$ with competitve $\mathrm{L}_{x}$ models namely: the $\mathrm{EL}_{x}$ model (Gupta et al., 1998), the gamma Lomax $\left(\mathrm{GaL}_{x}\right)$ model (Cordeiro et al., 2015), the beta Lomax $\left(\mathrm{BL}_{x}\right)$ model (Lemonte and Cordeiro, 2013) and $\mathrm{L}_{x}$ model. The CDFs of these distributions are, respectively, given by (for $x>0$ and $\alpha, \beta, \lambda, a>0$ ):

$$
\begin{aligned}
& F_{\alpha, \beta, \lambda}(x)=\left[1-\left(1+x \beta^{-1}\right)^{-\lambda}\right]^{\alpha} \\
& F_{\alpha, \beta, \lambda}(x)=\Gamma^{-1}(\alpha) \Gamma\left(\alpha ; \lambda \log \left[1+x \beta^{-1}\right]\right)
\end{aligned}
$$

and:

$$
F_{\alpha, \beta, \theta, \lambda}(x)=\frac{1}{B(\alpha, \theta)} B\left(1-\left(1+x \beta^{-1}\right)^{-\lambda} ; \alpha, \theta\right)
$$

where, $\Gamma(\cdot)$ is the gamma function, $\Gamma(\cdot ;)$ is the incomplete gamma function, $B(\cdot, \cdot)$ is the complete beta function and $B(\cdot ; \cdot, \cdot)$ is the incomplete beta function.

\section{Data Set I}

The first real data set represents the data on failure times of 84 aircraft wind-shield given in Murthy et al. (2004). The data are: $0.0400,1.8660,2.3850,3.4430$, $0.3010,1.8760,2.4810,3,4.035,1.281,2.0850$, $1.98100,2.6610,4.449,1.6190,2.224,2.890,4.121$, $1.3030,2.089,3.7790,3.4670,0.3090,1.8990,2.610$, $3.4780,0.5570,1.248,2.0100,2.688,3.9240,1.2810$, 2.038, 2.820, 2.902, 4.167, 1.4320, 2.097, 2.934, 4.2400, $0.94300,1.9120,2.632,3.5950,1.0700,1.91400$, 2.2230, 3.1140, 1.9110, 2.6250, 3.5780, 3.699, 1.12400, $2.6460,1.480,2.135,1.5060,2.190,3.000,4.3050$, $1.568,2.1940,2.962,4.2550,1.5050,2.1540,2.9640$, $4.2780,3.103,4.376,1.615,3.1170,4.485,1.652$, $2.2290,3.166,4.570,1.652,2.3000,3.344,4.602$, $1.7570,2.324,3.3760,4.6630$. The Total Time Test (TTT) plot (Aarset, 1987) for data set I is presented in

\begin{tabular}{|c|c|c|c|c|c|c|}
\hline \multirow[b]{2}{*}{$\mathrm{n}$} & \multicolumn{2}{|l|}{$\hat{\alpha}$} & \multicolumn{2}{|l|}{$\hat{\lambda}$} & \multicolumn{2}{|l|}{$\hat{\beta}$} \\
\hline & Bias & MSE & Bias & MSE & Bias & MSE \\
\hline 20 & 0.186 & 0.04 & -0.421 & 0.19 & 0.308 & 0.108 \\
\hline 50 & 0.183 & 0.036 & -0.426 & 0.186 & 0.304 & 0.097 \\
\hline 100 & 0.182 & 0.035 & -0.431 & 0.189 & 0.304 & 0.095 \\
\hline 150 & 0.183 & 0.034 & -0.429 & 0.185 & 0.304 & 0.094 \\
\hline 500 & 0.183 & 0.034 & -0.032 & 0.187 & 0.305 & 0.093 \\
\hline 1000 & 0.081 & 0.034 & -0.032 & 0.187 & 0.005 & 0.093 \\
\hline 20 & 0.519 & 0.052 & -0.975 & 0.992 & 1.91 & 0.907 \\
\hline 50 & 0.208 & 0.049 & -0.996 & 1.009 & 0.901 & 0.844 \\
\hline 100 & 0.117 & 0.049 & -0.995 & 0.997 & 0.906 & 0.36 \\
\hline 150 & 0.102 & 0.048 & -0.994 & 0.994 & 0.911 & 0.84 \\
\hline 500 & 0.008 & 0.048 & -0.547 & 1.001 & 0.833 & 0.911 \\
\hline 1000 & 0.008 & 0.048 & -0.1 & 0.001 & 0.91 & 0.83 \\
\hline
\end{tabular}
Fig. 3. From Fig. 3 we note that the empirical HRF of data sets is increasing.

Table 2: The MLEs and Bias and MSE values for the OLiELx 


\section{Data Set II}

The second real data set represents the data on service times of 63 aircraft windshield given in Murthy et al. (2004). The data are: $0.046,1.436,2.4640,4.8810$, $1.262, \quad 2.5430,2.592,0.140,1.492,2.600,0.150$, $0.2800,2.878,0.487,1.9630,2.950,0.6220,1.978$, $3.0030,0.389,1.9200,3.622,1.0850,2.163,0.9000$, $2.053,3.1020,1.580,2.670,0.248,1.7190,2.717$, $0.952,2.065,3.3040,0.9960,2.117,3.483,1.003$, $2.1370,3.500,1.0100,2.141,1.794,2.819,0.3130$, $1.915,2.820,3.665,1.0920,2.183,3.695,1.1520$, $2.2400,4.015,1.183,2.3410,4.628,1.2440,2.435$, $4.806,1.2490,5.140$. The TTT plot for data set II is presented in Fig. 4. From Fig. 4 we note that the empirical HRF of data sets is also increasing.

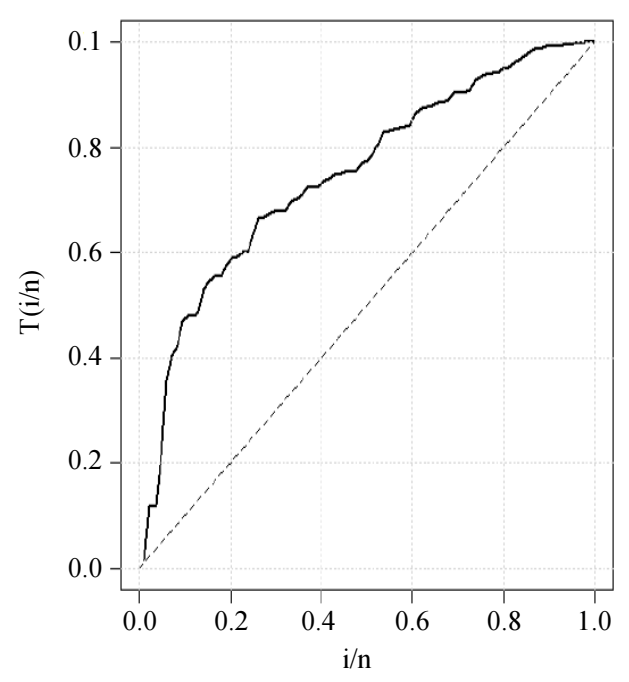

Fig. 3: TTT plots for data set I

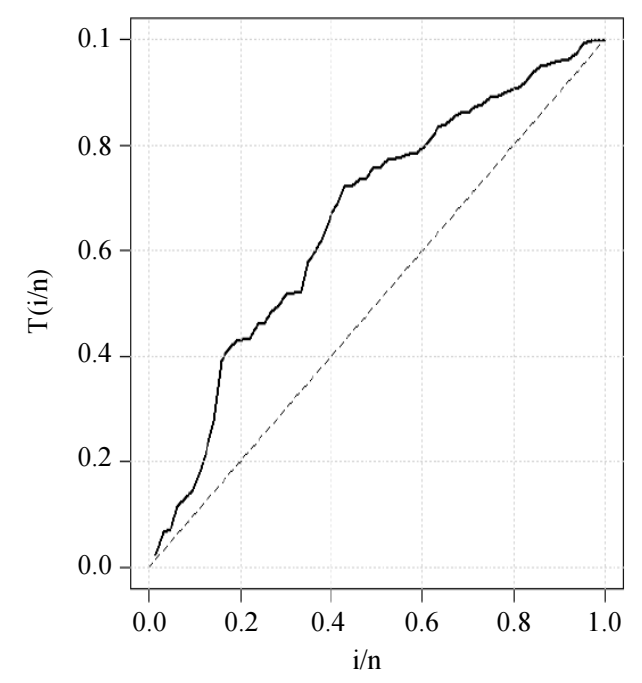

Fig. 4: TTT plots for data set II
These data sets were recently studied by Tahir et al. (2015). The unit for measurement is $1000 \mathrm{~h}$ for both data sets. In order to compare the distributions, the estimated log-likelihood values $\hat{\ell}$, Akaike Information Criteria (AIC), Cramer von Mises $\left(W^{*}\right)$ and Anderson-Darling $\left(A^{*}\right)$ goodness of fit statistics were calculated for all models. The statistics $W^{*}$ and $A^{*}$ are described in detail in Chen and Balakrishnan (1995). The $W^{*}$ and $A^{*}$ statistics are given by:

$$
W^{*}=(1+1 / 2 n)\left[\frac{1}{12 n}+\sum_{j=1}^{n} \omega_{j}\right],
$$

and:

$$
A^{*}=c^{(n)}\left(n+n^{-1} \sum_{j=1}^{n} c_{j}\right),
$$

where:

$$
\begin{aligned}
& \omega_{j}=\left[z_{i}-(2 j-1) /(2 n)\right]^{2}, \\
& c^{(n)}=1+\left[9 /\left(4 n^{2}\right)\right]+[3 /(4 n)],
\end{aligned}
$$

and:

$$
c_{j}=(2 j-1) \log \left[z_{i}\left(1-z_{n-j+1}\right)\right],
$$

where, $z_{i}=F\left(y_{j}\right)$ and the $y_{j}{ }^{\prime} s$ values are the ordered observations. The smaller these statistics are, the better the fit is. In general, it can be chosen as the best model which has the smaller values of the AIC, $W^{*}$ and $A^{*}$ statistics and the larger values of $\hat{\ell}$. The required computations are obtained by using the "maxLik" and "goftest" sub-routines in R-software. The analysis results of both these applications are listed in Table 3-6. These results show that the new distribution has the lowest AIC, $W^{*}$ and $A^{*}$ values and, has the biggest estimated $-\hat{\ell}$ among all the fitted models. Hence, it could be chosen as the best model under these criteria.

From Table 5 and 6, the proposed $\mathrm{OLiEL}_{x}$ lifetime model is much better than $\mathrm{GaL}_{x}, \mathrm{BL}_{x}, \mathrm{EL}_{x}$ and $\mathrm{L}_{x}$ models so the new model is a good alternative to these models in modeling aircraft windshield data.

Figure 5 gives the fitted PDF plot for the two data sets. Figure 6 gives the P-P plot for the two data sets. Figure 7 gives the fitted HRF plot for the two data sets. From Fig. 5-7 we note that the proposed model give adequate fit to the used data sets. 
The values in all tables are calculated using the $R$ program. We prove empirically that the new distribution provides better fits in two applications to real data sets

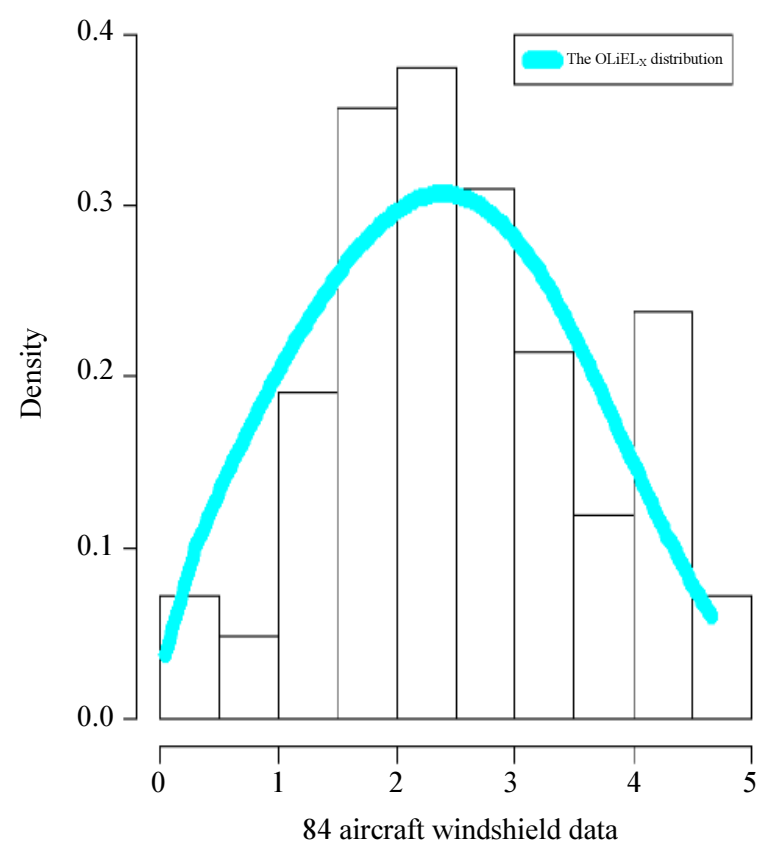

than other competitive extended $\mathrm{L}_{x}$ distributions. The OLiEL $_{x}$ distribution can be a good alternative for modeling failure times.

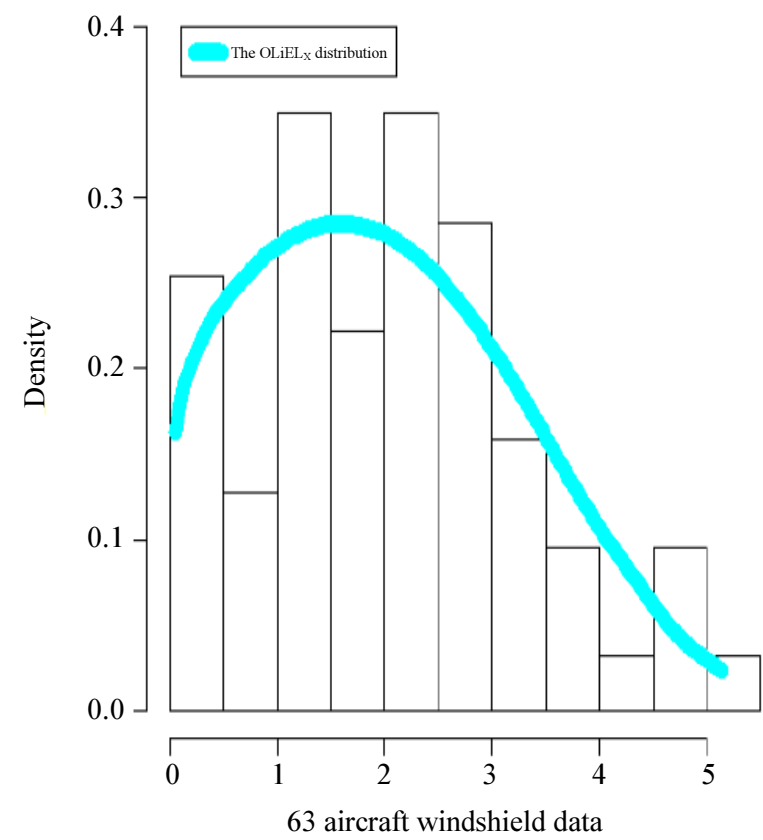

Fig. 5: The fitted PDF plot for data set I (left panel), the fitted PDF and CDF plot for data set II (right panel)
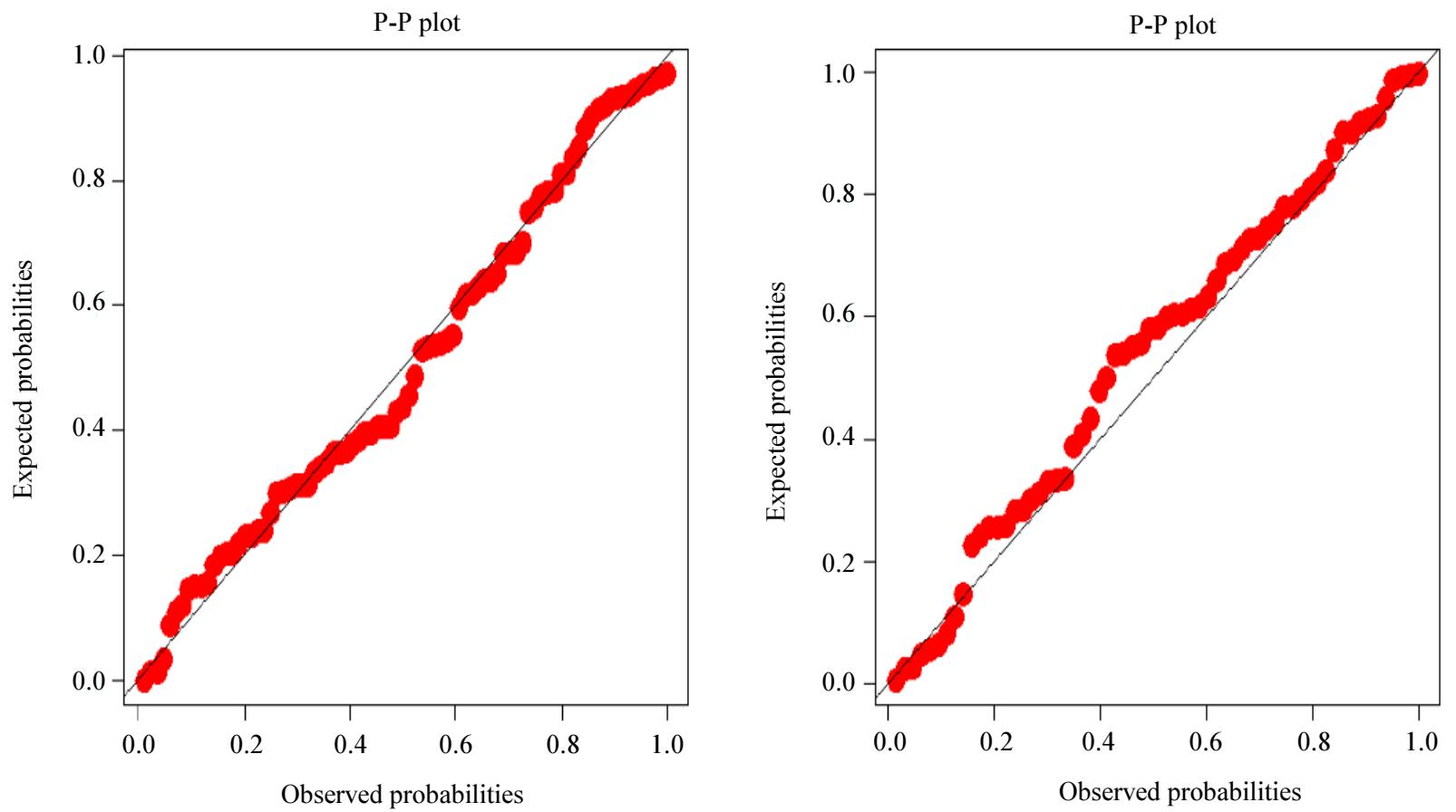

Fig. 6: The P-P plot for data set I (left panel), the P-P plot for data set II (right panel) 

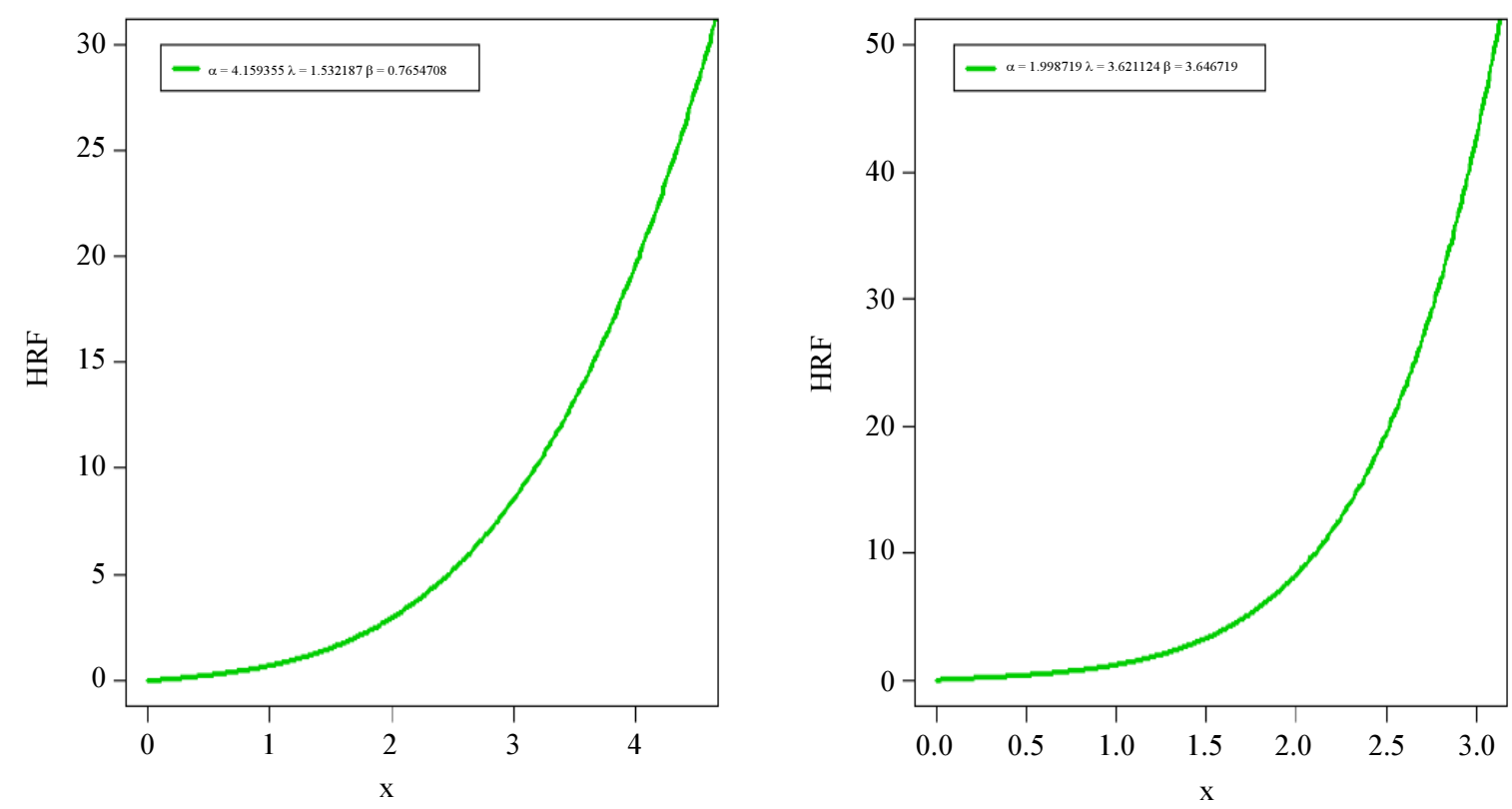

Fig. 7: The fitted HRF plot for data set I (left panel), the fitted PDF and HRF plot for data set II (right panel)

Table 3: MLEs, standard erros (in parentheses) for data set I

\begin{tabular}{lllll}
\hline Model & $\hat{\alpha}$ & $\hat{\beta}$ & $\hat{\theta}$ & $\hat{\lambda}$ \\
\hline OLiEL $_{x}$ & 0.1593 & 0.7655 & & 0.7322 \\
& $(0.3712)$ & $(0.04057)$ & $(1.7779)$ \\
$\mathrm{BL}_{x}$ & 3.6036 & 118.8374 & 33.638 & 4.8307 \\
& $(0.6187)$ & $(63.714)$ & $(9.238)$ & $(429.00)$ \\
$\mathrm{EL}_{x}$ & 3.6261 & 26257.6808 & 20074.51 \\
& $(0.6236)$ & $(99.742)$ & $(2041.83)$ \\
$\mathrm{GL}_{x}$ & 3.5876 & 37029 & 52001 \\
$\mathrm{~L}_{x}$ & $(0.5133)$ & $(81.1644)$ & $(7955)$ \\
& & 131789 & 51425 \\
\hline
\end{tabular}

Table 4: MLEs, standard erros (in parentheses) for data set II

\begin{tabular}{lllll}
\hline Model & $\hat{\alpha}$ & $\hat{\beta}$ & $\hat{\theta}$ & $\hat{\lambda}$ \\
\hline OLiEL $_{x}$ & 0.5987 & 0.6467 & & 1.624 \\
& $(0.39)$ & $(0.05)$ & 31.2595 & $(0.96)$ \\
$\mathrm{BL}_{x}$ & 1.9218 & 169.5800 & $(316.8)$ & $(50.585$ \\
& $(0.3185)$ & $(339.21)$ & & 22971.2 \\
$\mathrm{EL}_{x}$ & 1.9145 & 32881.9 & $(3209.5)$ \\
$\mathrm{GL}_{x}$ & $(0.3483)$ & $(162.22)$ & 35842.4 \\
$\mathrm{~L}_{x}$ & 1.9073 & 39197.6 & $(6945)$ \\
& $(0.3214)$ & $(151.653)$ & 99269 \\
\end{tabular}

Table 5: $-\hat{\ell}$ and goodness-of-fits statistics for data set I

\begin{tabular}{lllll}
\hline Model & $-\hat{\ell}$ & AIC & $W^{*}$ & $A^{*}$ \\
\hline $\mathrm{OLiEL}_{x}$ & 126.923 & 259.846 & 0.0907 & 0.0875 \\
$\mathrm{BL}_{x}$ & 138.718 & 285.4354 & 1.4084 & 0.1680 \\
$\mathrm{EL}_{x}$ & 141.3997 & 288.7994 & 1.7435 & 0.2194 \\
$\mathrm{GL}_{x}$ & 138.404 & 282.809 & 1.3667 & 0.1619 \\
$\mathrm{~L}_{x}$ & 164.990 & 333.9767 & 1.3976 & 0.1665 \\
\hline
\end{tabular}


Table 6: $-\hat{\ell}$ and goodness-of-fits statistics for data set II.

\begin{tabular}{lllll}
\hline Model & $-\hat{\ell}$ & AIC & $W^{*}$ & $A^{*}$ \\
\hline OLiEL $_{x}$ & $\mathbf{9 8 . 1 0 2 9 4}$ & $\mathbf{2 0 2 . 2 0 5 9}$ & $\mathbf{0 . 4 9 8 9}$ & $\mathbf{0 . 0 4 8 8}$ \\
$\mathrm{BL}_{x}$ & 102.9611 & 213.9223 & 1.1336 & 0.1872 \\
$\mathrm{EL}_{x}$ & 103.5468 & 213.9223 & 1.2331 & 0.2037 \\
$\mathrm{GL}_{x}$ & 102.8333 & 211.6664 & 1.1121 & 0.2038 \\
$\mathrm{~L}_{x}$ & 109.2988 & 222.5976 & 1.1265 & 0.1861 \\
\hline
\end{tabular}

\section{Conclusion}

In this article, we introduce and study a new alternative Lomax model. The maximum likelihood estimation method is used to estimate the unknown model parameters. We show empirically the importance and wide flexibility of the new model in modeling two types of failure times data sets. This model is much better than gamma Lomax, exponentiated Lomax, beta Lomax and Lomax models so the new model is a good alternative to these models. We hope that the new model will attract wider applications in engineering, reliability and other areas of research.

\section{Acknowledgment}

The author gratefully acknowledge with thanks the very thoughtful and constructive comments and suggestions of the Editor-in-Chief and the reviewers which resulted in much improved paper.

\section{Ethics}

The author declares that there is no conflict of interests regarding the publication of this article.

\section{References}

Aarset, M.V., 1987. How to identify a bathtub hazard rate. IEEE Trans. Reliability, 36: 106-108. DOI: 10.1109/TR.1987.5222310

Afify, A.Z., Z.M. Nofal, H.M. Yousof, Y.M. El Gebaly and N.S. Butt, 2015. The transmuted Weibull Lomax distribution: Properties and application. Pak. J. Stat. Oper. Res., 11: 135-152. DOI: $10.18187 /$ pjsor.v11i1.956

Atkinson, A.B. and A.J. Harrison, 1978. Distribution of Personal Wealth in Britain. 1st Edn., Cambridge University Press, Cambridge, ISBN-10: 0608156906, pp: 344.

Chen, G. and N. Balakrishnan, 1995. A general purpose approximate goodness-of-fit test. J. Quality Technol., 27: 154-161.

DOI: $10.1080 / 00224065.1995 .11979578$
Gupta, R.C., P.L. Gupta and R.D. Gupta, 1998. Modeling failure time data by Lehman alternatives. Commun. Stat. Theory Meth., 27: 887-904. DOI: $10.1080 / 03610929808832134$

Cordeiro, G.M., E.M. Ortega and B.V. Popovic, 2015. The gamma-Lomax distribution. J. Stat. Comput. Simulat., 85: 305-319. DOI: $10.1080 / 00949655.2013 .822869$

Glanzel, W., 2008. On some new bibliometric applications of statistics related to the h-index. Scientometrics, 77: 187-196. DOI: $10.1007 / \mathrm{s} 11192-007-1989-0$

Harris, C.M., 1968. The Pareto distribution as a queue service descipline. Operat. Res., 16: 307-313. DOI: 10.1287/opre.16.2.307

Hassan, A.S. and A.S. Al-Ghamdi, 2009. Optimum step stress accelerated life testing for Lomax distibution. J. Applied Sci. Res., 5: 2153-2164.

Lemonte, A.J. and G.M. Cordeiro, 2013. An extended Lomax distribution. Statistics, 47: 800-816. DOI: 10.1080/02331888.2011.568119

Lomax, K.S., 1954. Business failures: Another example of the analysis of failure data. J. Am. Stat. Assoc., 49: 847-852. DOI: $10.2307 / 2281544$

Murthy, D.N.P., M. Xie and R. Jiang, 2004. Weibull Models. 1st Edn., Wiley, Hoboken, ISBN-10: 0471473278, pp: 408.

Silva, F.S., A. Percontini, E. de Brito, M.W. Ramos, R. Venancio and G.M. Cordeiro, 2017. The odd Lindley-G family of distributions. Austrian J. Stat., 46: 65-87. DOI: 10.17713/ajs.v46i1.222

Tahir, M.H., G.M. Cordeiro, M. Mansoor and M. Zubair, 2015. The Weibull-Lomax distribution: Properties and applications. Hacettepe J. Math. Stat., 44: 461-480.

Yousof, H.M., A.Z. Afify, G.G. Hamedani and G. Aryal, 2017. The Burr $X$ generator of distributions for lifetime data. J. Stat. Theory Applic., 16: 288-305. DOI: $10.2991 /$ jsta.2017.16.3.2 